\title{
Asociación de Partición Hepática y Ligadura Portal para Hepatectomía por Etapas (ALPPS)
}

\author{
Associating Liver Partition and Portal Vein Ligation for Staged Hepatectomy (ALPPS)
}

\author{
Carlos Manterola ${ }^{1,2} \&$ Tamara Otzen ${ }^{2,3}$
}

\begin{abstract}
MANTEROLA, C. \& OTZEN, T. Asociación de partición hepática y ligadura portal para hepatectomía por etapas (ALPPS). Int. J. Morphol., 35(3):1083-1090, 2017.

RESUMEN: La disfunción hepática postoperatoria del remanente hepático que ocurre en pacientes sometidos a grandes resecciones hepáticas, es un problema complejo y temido, dado su pronóstico incierto. La asociación de partición hepática y ligadura portal para hepatectomía por etapas (ALPPS), es un enfoque novedoso para pacientes portadores de enfermedad hepática oncológica que anteriormente eran considerados "no resecables". El procedimiento se realiza en dos etapas. La primera, comprende la ligadura de la rama derecha de la vena porta. Luego, se realiza la transección del parénquima hepático; incluyendo o no, la sección y ligadura de la vena hepática media. A continuación se empaqueta el hígado tumoral en una bolsa de polietileno y el abdomen es cerrado. La segunda etapa, se realiza 7 a 15 días después. Una vez abierto el abdomen, se retira la bolsa de polietileno; se ligan y seccionan la arteria, el conducto biliar y la vena hepática derechos; y se elimina el hígado tumoral. Pueden instalarse drenes y se procede al cierre de la laparotomía. La técnica ALPPS puede permitir entonces, la resección curativa de hígados tumorales en pacientes con lesiones considerados previamente como no resecables. El objetivo de este artículo fue describir las indicaciones y aspectos técnicos del ALPPS a propósito del primer caso realizado en nuestra ciudad, en una paciente de 47 años con un cáncer de vesícula biliar avanzado y metástasis bilobares.
\end{abstract}

PALABRAS CLAVE: Neoplasia hepática; Metástasis hepáticas; Hígado metastásico; Hepatectomías; Trisegmentectomía derecha; Cáncer de la vesícula biliar; Insuficiencia hepática aguda; Ligadura de la vena porta; Regeneración hepática; ALPPS.

\section{INTRODUCTION}

Las resecciones hepáticas constituyen el único tratamiento con intención curativa en pacientes con enfermedad hepática maligna (primaria o secundaria); sin embargo, la probabilidad de resección curativa puede estar restringida al remanente hepático, y por ende a la eventual insuficiencia hepática postoperatoria; que representa la complicación más grave en este tipo de procedimientos (Margarit, 2005). Esto, debido a que hay evidencia que para evitarla, se requiere al menos un $25 \%$ del volumen hepático total en hígados sanos y un $40 \%$ en hígados enfermos, o que hayan recibido quimioterapia (Rahbari et al., 2011); lo que suele ser habitual de observar en este tipo de pacientes.

Por ello, se han desarrollado una serie de estrategias preventivas para este problema; desde algunas preoperatorias como la descontaminación intestinal selectiva para reducir la translocación bacteriana postoperatoria favorecida por el íleo postoperatorio, la insuficiencia hepática y la hipertensión por- tal; hasta otras intra y postoperatorias como la prescindencia de la utilización de fármacos hepatotóxicos, el pinzamiento intermitente del pedículo hepático, que puede remedar un precondicionamiento isquémico (Clavien et al., 2000); mantener una correcta oxigenación tisular y una hemodinamia adecuada, de modo tal que el hígado remanente logre mantener sus funciones y comience el proceso de regeneración hepática. Junto a lo cual es fundamental una profilaxis antibiótica; una correcta nutrición postoperatoria, que colaborará en la regeneración hepática, etc. (Mann et al., 2001; Yigitler et al., 2003).

Sin embargo, a pesar de todo lo anteriormente expuesto, es sabido que resecciones hepáticas extensas, pueden dejar un remanente hepático insuficiente; lo que ha movido a diversos grupos a desarrollar alternativas técnicas orientadas a producir una hipertrofia del remanente hepático; entre las que destacan la embolización portal percutánea (Makuuchi et

\footnotetext{
${ }^{1}$ Departamento de Cirugía, Universidad de La Frontera, Temuco, Chile.

${ }^{2}$ Centro de Estudios Morfológicos y Quirúrgicos (CEMyQ), Universidad de La Frontera, Temuco, Chile.

${ }^{3}$ Facultad de Ciencias de la Salud, Universidad de Tarapacá, Arica, Chile.

Financiamiento Parcialmente financiado por proyecto DI16-0119 de la Dirección de Investigación Universidad de La Frontera, Chile.
} 
al., 1990) y la ligadura portal intraoperatoria, con o sin infusión de quimioterápicos (Gerard et al., 1991; Ribero et al., 2007; Abulkhir et al., 2008; Capussotti et al., 2008). Sin embargo, estas técnicas de oclusión portal, producen una hipertrofia tardía, que puede lograrse 4 a 8 semanas después; lo que influye en una progresión tumoral (Elias et al., 1999; de Graaf et al., 2009; Björnsson et al., 2016a).

En 2011 se describió el "associating liver partition and portal ligation for staged hepatectomy" (ALPPS); alternativa innovadora, que permite lograr resultados similares a los anteriores, pero en tiempos menores (Baumgart et al., 2011); y a partir de 2012, comenzaron a publicarse las primeras series de ALPPS (Schnitzbauer et al., 2012; Torres et al., 2013; De Santibañes \& Clavien, 2012; Robles et al., 2012) (Figs. 1-3).

Esta alternativa, representa pues una interesante opción para reducir la insuficiencia hepática postoperatoria, permitiendo amplias resecciones en pacientes con enfermedad localmente avanzada (especialmente por metástasis de carcinoma colorrectal (Donati et al., 2013), considerados previamente no resecables debido a un remanente hepático insuficiente (Li et al., 2013; Knoefel et al., 2013; Alvarez et al., 2013; Oldhafer et al., 2014; Fukami et al., 2014).

Este procedimiento se basa en que el hígado tumoral privado de flujo portal, actúa como "hígado auxiliar", mientras se produce la hipertrofia del hígado remanente. Por otra parte, al no existir flujo cruzado intraparenquimatoso, debido a la transección hepática; se maximiza el aumento de volumen en el remanente hepático (Donati et al.; Zhang et al., 2014).

El primer caso publicado en Chile, corresponde a una paciente con metástasis hepáticas múltiples originadas en un cáncer de colon, en la que se logró un buen resultado, con un seguimiento a dos años (Uribe et al., 2015).

El objetivo de este artículo fue describir las indicaciones y aspectos técnicos del ALPPS a propósito del primer caso realizado en nuestra ciudad, en una paciente de 47 años con un cáncer de vesícula biliar avanzado, con metástasis bilobares (Fig. 4); que hasta la fecha de la escritura de este manuscrito es el $8^{\circ}$ caso de este tipo en el que se aplicó esta técnica.

\section{ASPECTOS TÉCNICOS}

Vía de acceso: Laparotomía subcostal bilateral o laparotomía en "J" a derecha. Exploración abdominal completa para asegurar que no existe carcinomatosis peritoneal (Ratti et al., 2014; Chan et al., 2015); y valorización del hígado tumoral y del remanente que quedará después de la resección (Fig. 1).

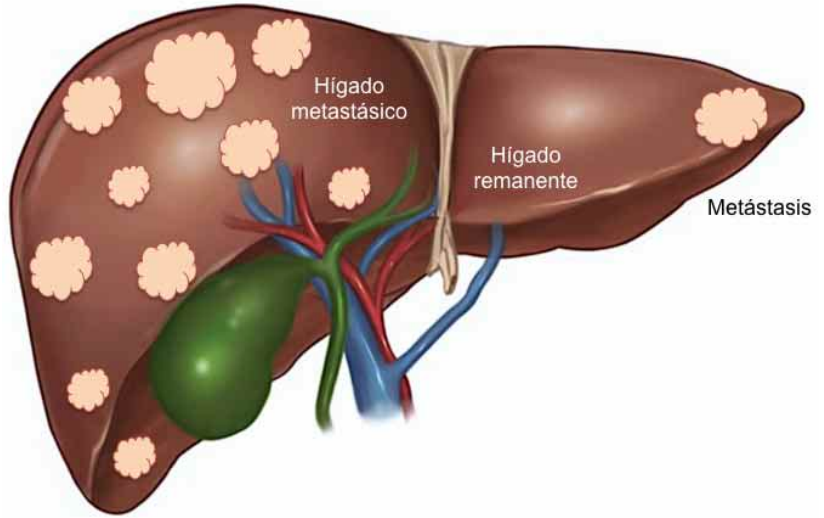

Fig. 1. Hígado metastásico con múltiples lesiones bilobares, que dejan un escaso porcentaje de víscera indemne o remanente. Los tumores se destacan en color anaranjado suave.

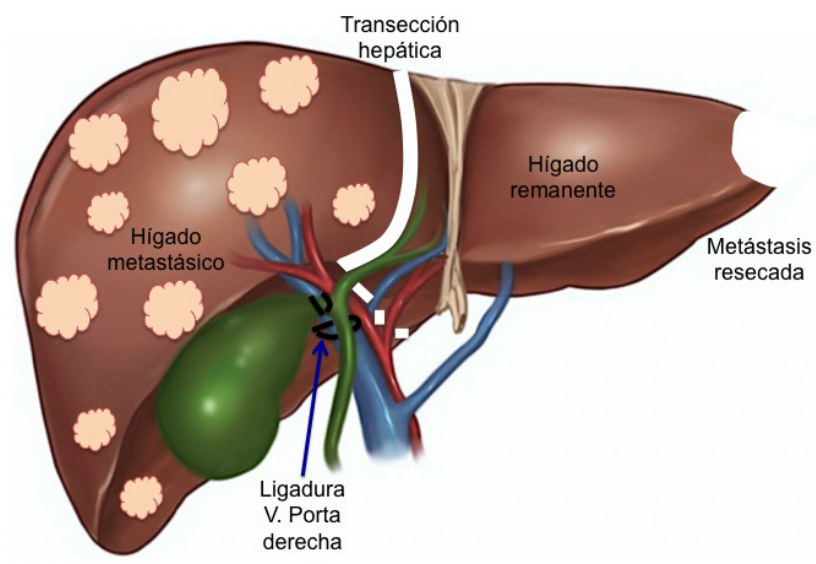

Fig. 2. Se aprecia la división in situ entre el sector lateral izquierdo y parte del segmento IV con ligadura de la vena porta derecha. La eliminación del tumor en el sector lateral izquierdo fue también realizada en esta etapa.

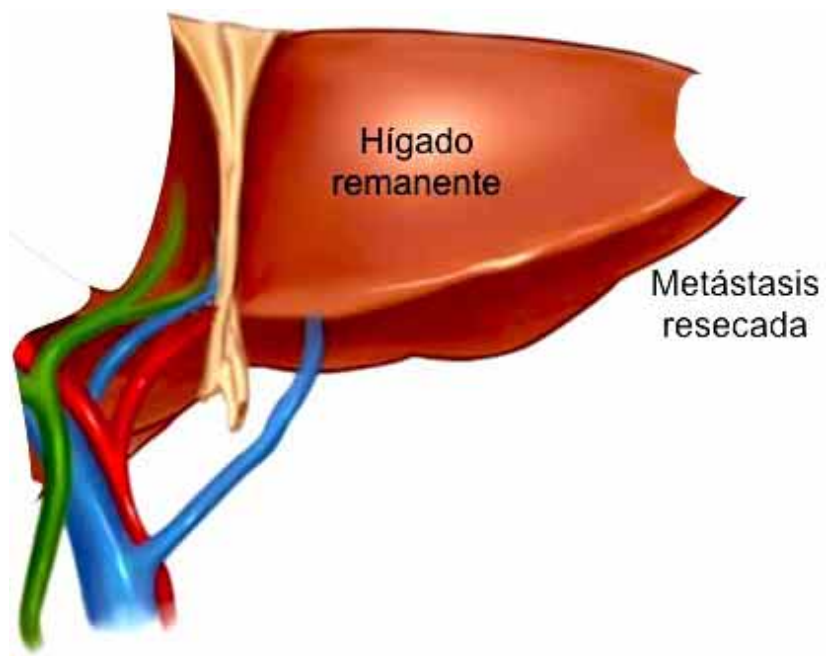

Fig. 3. Completado la segunda fase de ALPPS. Se evidencia el hígado remanente con su pedículo vascular y biliar; y la metástasis lateral izquierda resecada. 


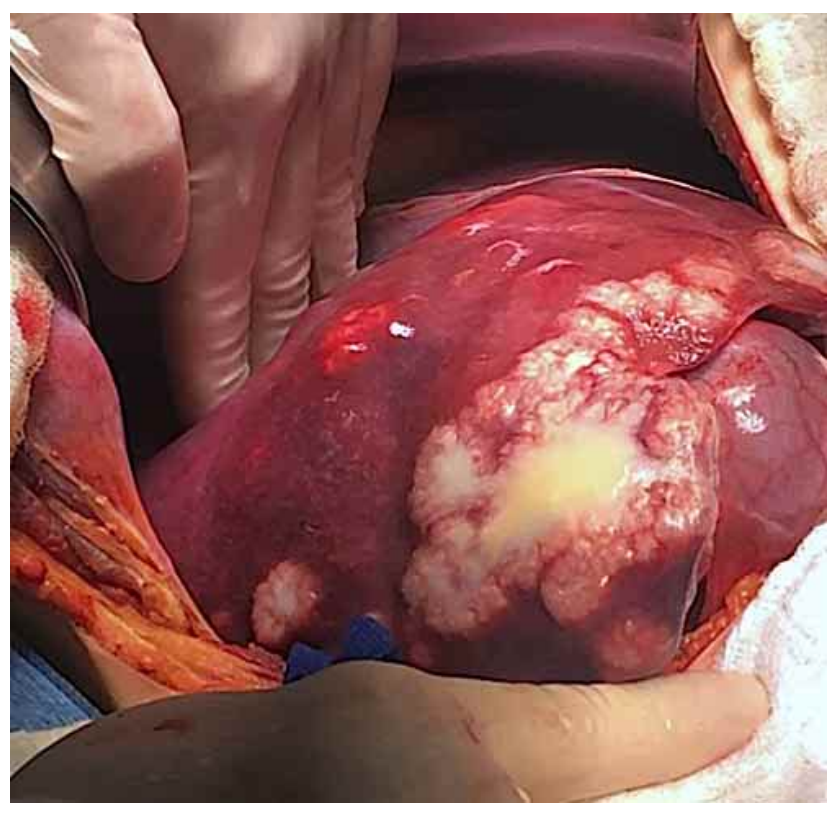

Fig. 4. Tomografía axial computarizada con contraste, en corte sagital (pre y postoperatoria). A la izquierda el hígado tumoral, con linfonodos tumorales que invaden el pedículo hepático y un pequeño remanente hepático en el que se aprecia una lesión metastásica lateral izquierda. A la derecha, visión postoperatoria del remanente hepático hipertrofiado desplazado al hipocondrio derecho, libre de lesiones tumorales.

Ultrasonografía intraoperatoria: Es recomendable realizar una detallada exploración del hígado, mediante ultrasonido, pues permite verificar hallazgos no observados en las imágenes preoperatorias, así como también, delimitar mejor el sitio de sección y los elementos vasculares y biliares que se han de tomar en consideración al momento de la transección (Sala et al., 2012).

Gestos quirúrgicos preliminares: Movilización total del hígado, seccionando ligamentos redondo, suspensorio, coronales y triangulares bilaterales. Disección del pedículo portal, esqueletizándolo y extirpando el tejido linfonodal (si aplica, dependiendo del origen de la enfermedad tumoral); y secciónligadura de venas hepáticas accesorias (Ratti et al.; Bertens et al., 2015; Buac et al., 2016).

Ligadura portal y transección parenquimatosa: Una vez apropiadamente disecada, la rama portal derecha es ligada y seccionada, preservando la arteria y el conducto hepático derecho. Además, se ligan y seccionan la arteria y conducto cístico, removiendo la vesícula biliar (Ettorre et al., 2015). Una vez realizado este gesto, se procede a la partición total o parcial (ALTPS; Robles Campos et al. 2014) del parénquima hepático. Existe una tendencia a preservar la vena suprahepática media, de modo tal de evitar la congestión de los segmentos IV, V y VIII (Bertens et al.) (Fig. 2).
Empaquetamiento hepático: Una vez finalizada la transección total o parcial (ALTPS), el hígado tumoral es envuelto en una bolsa de polietileno para evitar la formación de adherencias y recolectar eventuales fluidos serohemáticos y biliosos (Bertens et al.; Chan et al.; Ettorre et al.; Cai et al., 2016). Sin embargo, existe evidencia que sustenta que la congestión hepática es importante para una regeneración más rápida del hígado remanente posterior a la realización de un ALPPS (Kawaguchi et al., 2016).

Segunda etapa: Después de algunos días (entre 7 y 14), se programa la segunda etapa, que consiste en la remoción del hígado tumoral previa ligadura y sección de la arteria y conducto hepático derecho y de la vena suprahepática derecha (y media si no se ligó en la primera etapa) (Bertens et al.; Chan et al.; Uribe et al.) (Fig. 3).

Variantes técnicas: Se han descrito una serie de variantes, tanto técnicas como relacionadas con el remanente hepático. Por ejemplo, existen evidencia tanto de ALPPS derecho como izquierdo; monosegmentario, dejando un remanente hepático de un solo segmento (Rochet et al., 2014); el ALTS descrito por un grupo español, que en vez de transección, aplica un torniquete oclusivo (Robles Campos et al.); la modificación sugerida por un grupo japonés, con el que plantean que se ahorran pedículos portales en el plano de transacción, manteniendo el suministro de sangre al preservar al máximo los pedículos portales (Tanaka et al., 2017); etc.

\section{INDICACIONES Y CONTRAINDICACIONES}

Las indicaciones de ALPPS y ALTPS, incluyen pacientes que requieren de hepatectomía derecha extendida debido a tumores hepáticos primarios marginalmente resecables o localmente avanzados (colangiocarcinoma intrahepático y perihiliar, y carcinoma hepatocelular), y por metástasis múltiples de origen colorrectal. Sin embargo, existen otras indicaciones mucho menos frecuentes como metástasis de tumores de Wilms, ovario, cérvix uterino, estómago. GISTs y melanoma (Nadalin et al., 2014; Schadde et al., 2014a; Ratti et al.; Rochet et al.; Zhang et al., 2014; Hasselgren et al., 2015; Lang et al., 2015; Truant et al., 2015; Vivarelli et al., 2015; Buac et al.; Björnsson et al., 2016a; Røsok et al., 2016); todo lo cual, debe estar en relación con la existencia de un hígado remanente menor de $25 \%$ a $30 \%$ (hígados sanos) y menor de $40 \%$ (hígados colestásicos, esteatósicos, con fibrosis, o con cambios patológicos secundarios a quimioterapia (Nadalin et al.; Ratti et al.; Zhang et al.; Buac et al.).

Existe además, evidencia (aunque escasa), de su utilización en pacientes con cáncer de vesícula biliar metastásico, que hasta el momento es de sólo 8 casos publicados (Ratti et al.; Hasselgren et al.; Kremer et al., 2015; Truant et al.) 
Por otra parte, el ALPPS estaría contraindicado en pacientes con hígado tumoral no resecable en el remanente hepático, existencia de metástasis extra hepáticas no resecables, hipertensión portal grave; así como también, cuando existen contraindicaciones medicas y anestésicas para una cirugía mayor (Zhang et al.). Además, se han considerado como contraindicación relativa edad mayor de 70 años y pacientes con bajo performance status (Bertens et al.).

\section{RESULTADOS}

A pesar de llevar 6 años de historia, el número de casos publicados de pacientes con ALPPS es aún escaso; con un número total que debe superar en poco los 700 casos a nivel mundial. Se trata mayoritariamente de series pequeñas, inferiores a 50 pacientes (con varias de ellas menores de 20 casos); con excepción de una que agrupa varios centros, llegando a 202 casos (Schadde et al., 2014b) (Tabla I). Resultados primarios: Existe evidencia que tras un intervalo de 9 a 14 días entre las dos etapas, se produce una hipertrofia del remanente de $61 \%$ a $93 \%$ (Schnitzbauer et al.; Alvarez et al, 2013; Li et al.; Knoefel et al.; Torres et al.; Nadalin et al.; Oldhafer et al.; Ratti et al.; Robles Campos et al.; Schadde et al., 2014a; Hernandez-Alejandro et al., 2015) (Figs. 5 y 6). Por otra parte, existe evidencia que cerca del $100 \%$ de los pacientes logran completar las dos eta- pas y que en entre el $86 \%$ y el $100 \%$ se obtuvieron resecciones R0 (Schnitzbauer et al.; Alvarez et al, 2013; Knoefel et al.; Li et al.; Torres et al.; Nadalin et al.; Oldhafer et al.; Ratti et al.; Robles Campos et al.; Schadde et al., 2014b; Hernandez-Alejandro et al.).

Morbilidad postoperatoria: Existe evidencia que la morbilidad postoperatoria total es muy alta; se mencionan cifras de 53,0 \% a 91,0 \% (Nadalin et al.; Rochet et al.; Lang et al.; Adam et al., 2016; Björnsson et al., 2016b); gran parte de la cual es $\geq$ a grado IIIB de Clavien-Dindo (Clavien et al., 2009; Nadalin et al.; Hernandez-Alejandro et al.; Tanaka et al., 2015). Cuando se requiere realizar reconstrucciones biliares, por colangiocarcinoma hiliares, la morbilidad puede incluso ser mayor (Schnitzbauer et al.; Li et al.; Nadalin et al.). Se ha descrito también que la resección de metástasis hepáticas no-colorrectales sería un factor predictor independiente de mayor gravedad de complicaciones (Schadde et al., 2014b).

Mortalidad postoperatoria: Existe evidencia que la mortalidad postoperatoria puede llegar hasta un 29,0 \% ( $\mathrm{Li}$ et al.; Nadalin et al.; Cai et al.). Pero, además, se ha reportado mortalidad tardía (posterior a los 90 días), la que puede llegar a 12,8 \% (Schnitzbauer et al.; Rochet et al.; Lang et al.; Tanaka et al., 2015; Björnsson et al., 2016b). Últimamente, se desarrolló un escore predictivo de riesgo de mortalidad postoperatorio inmediata y a 90 días, con base en un análisis multivariado (Linecker et al., 2016).

Tabla I. Resumen de resultados reportados de alpps.

\begin{tabular}{|c|c|c|c|c|c|c|}
\hline Autor, fecha & $\begin{array}{c}\mathbf{N}^{\circ} \\
\text { casos }\end{array}$ & $\begin{array}{c}\text { Aumento } \\
\text { volumen } \\
(\%)\end{array}$ & $\begin{array}{c}\text { Hospitalización } \\
\text { (días) }\end{array}$ & $\begin{array}{c}\text { Morbilidad } \\
\text { (\%) }\end{array}$ & $\begin{array}{l}\text { Mortalidad } \\
(\%)\end{array}$ & $\begin{array}{c}\text { Recurrencia } \\
(\%)\end{array}$ \\
\hline Schnitzbauer et al., 2012 & 25 & 74 & 12,0 & 60,0 & 12,0 & NR \\
\hline Sala et al., 2012 & 10 & 82 & 16,0 & 40,0 & 0,0 & 20,0 \\
\hline Torres et al., 2013 & 39 & 83 & 13,0 & 59,0 & 13,0 & NR \\
\hline Li et al., 2013 & 9 & 87 & 22,0 & 66,0 & 22,0 & NR \\
\hline Oldhafer et al., 2014 & 7 & 65 & 16,6 & 86,0 & 0,0 & 86,0 \\
\hline Nadalin et al., 2014 & 15 & 87 & 29,0 & 67,0 & 29,0 & 40,0 \\
\hline Ratti et al., 2014 & 12 & 47 & 8,3 & 83,0 & 8,3 & 27,0 \\
\hline Robles Campos et al., 2014 & 22 & 61 & 9,0 & 63,0 & 9,0 & 5,0 \\
\hline Schadde et al., 2014a & 48 & 77 & 15,0 & 73,0 & 15,0 & NR \\
\hline Schadde et al., 2014b & 202 & 86 & 9,0 & 68,0 & 9,0 & NR \\
\hline Alvarez et al., 2015 & 30 & 90 & 6,6 & 53,0 & 6,6 & 40,0 \\
\hline Chan et al., 2015 & 13 & 53 & 7,7 & 15,3 & 7,7 & NR \\
\hline Hernandez-Alejandro et al., 2015 & 14 & 93 & 23,0 & 36,0 & 0,0 & 14,3 \\
\hline Lang et al., 2015 & 16 & 86 & 12,5 & 64,0 & 12,5 & 56,0 \\
\hline Tanaka et al., 2015 & 11 & 54 & 11,0 & 64,0 & 9,0 & NR \\
\hline Truant et al., 2015 & 62 & 48 & 12,9 & 80,6 & 12,9 & NR \\
\hline Adam et al., 2016 & 17 & NR & NR & 59,0 & 0,0 & 47,1 \\
\hline Bjömsson et al., 2016b & 23 & NR & 9,0 & 91,0 & 4,5 & 34,8 \\
\hline Røsok et al., 2016 & 36 & 67 & NR & 92,0 & 2,8 & NR \\
\hline Tanaka et al., 2017 & 5 & NR & NR & 80,0 & 0,0 & NR \\
\hline
\end{tabular}




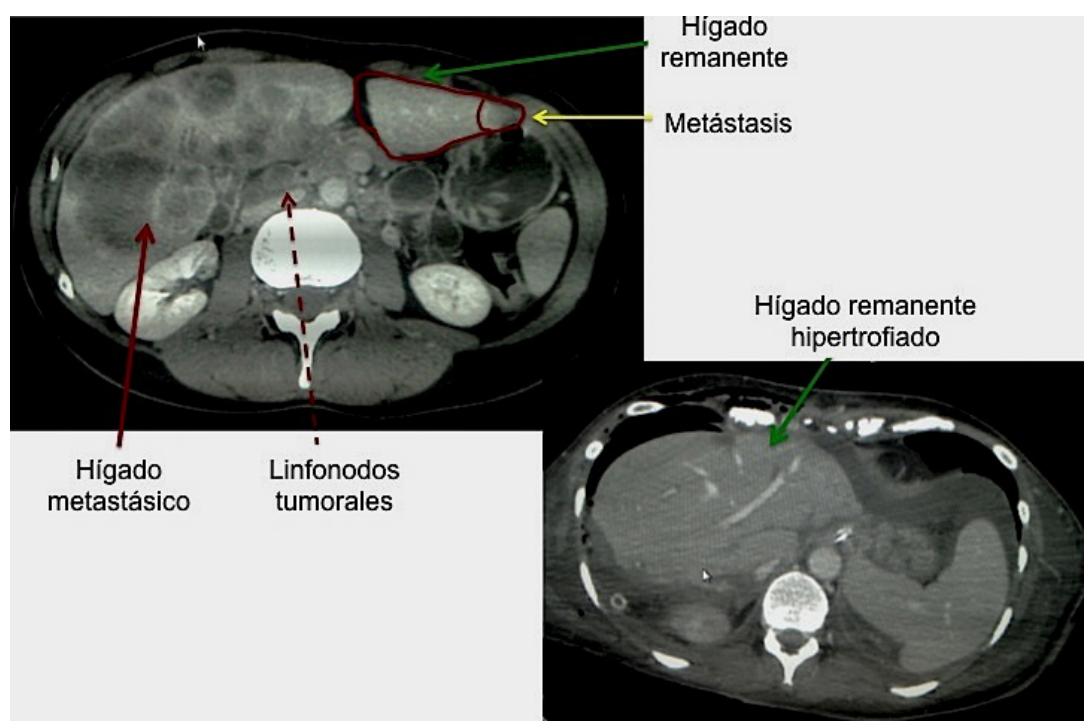

Fig. 5: Tomografía axial computarizada con contraste, en corte axial (pre y postoperatoria). A la izquierda el hígado tumoral con un pequeño remanente hepático. A derecha, visión postoperatoria del remanente hepático hipertrofiado desplazado al hipocondrio derecho, libre de lesiones tumorales.
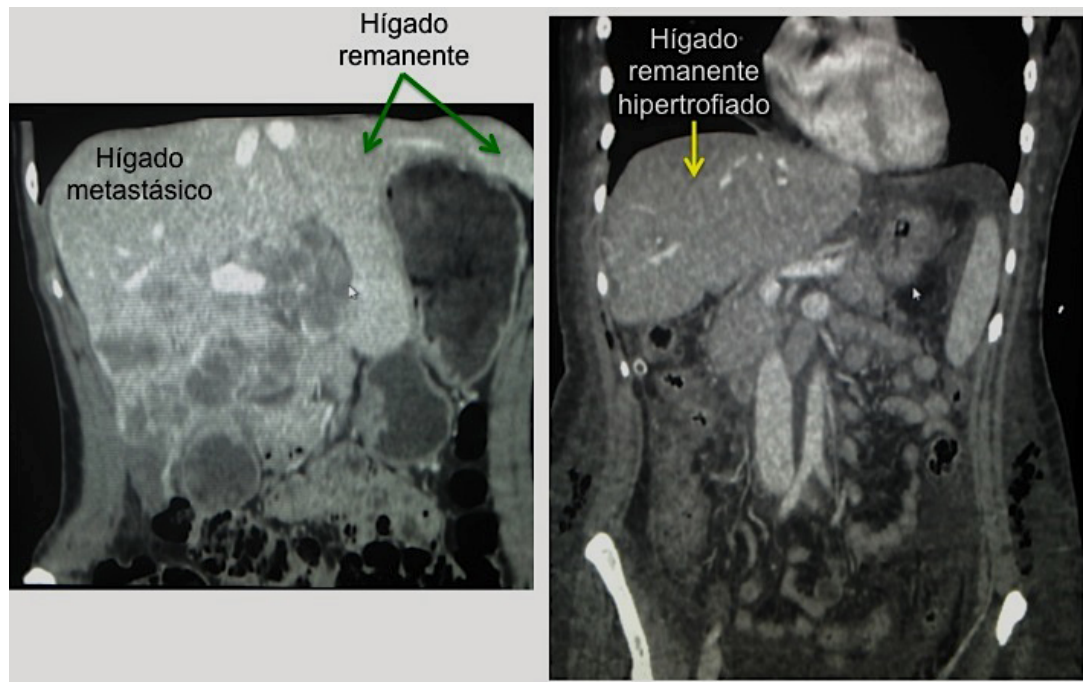

Fig. 6: Visión intraoperatoria de un hígado metastásico con origen en un cáncer de vesícula biliar avanzado, con múltiples lesiones bilobares.

Comparación con la hepatectomía convencional de dos etapas: Existe evidencia escasa respecto de este punto. Lo que se menciona es que ALPPS es una alternativa que ofrece una mejor oportunidad de una resección completa respecto de la técnica convencional. Existen algunos estudios que han comunicado que no habría diferencias en la magnitud de la hipertrofia ni en la morbilidad postoperatoria (Shindoh et al., 2013; Schadde et al., 2014b; Alvarez et al., 2015); aunque sí en una mayor mortalidad postoperatoria. La hipertrofia del remanente con ALPPS se ha verificado en algunas series sobre el $80 \%$ (Sala et al.; Torres et al.; Li et al., 2013; Nadalin et al.; Schadde et al., 2014b; Alvarez et al., 2015; Hernandez-Alejandro et al.; Lang et al.); lo que puede estar asociado a diversas variables, como la co-existencia de co-morbilidades, las características del parénquima hepático, quimioterapia adyuvante, origen y extensión de la enfer- medad metastásica, uso de clampeo intermitente, tiempo entre una etapa y otra, etc.

Seguimiento a largo plazo: A medida que ha ido aumentando el tiempo transcurrido desde la primera publicación, los datos referentes a tiempos de seguimiento se han ido incrementando. De modo tal, que hay reportes de supervivencia libre de enfermedad de $73,0 \%$ a $95,0 \%$ para una mediana de seguimiento de 6 meses; lo que disminuye a entre $46,0 \%$ y 60,0\% a 12 meses (Alvarez et al, 2013; Schadde et al., 2014a; Schadde et al., 2014b; Hernandez-Alejandro et al.). Para casos específicos de metástasis colorrectales, la supervivencia libre de enfermedad reportada a 12 y 24 meses es de $59,0 \%$ y $41,0 \%$ respectivamente (Schadde et al., 2014b). Por otra parte, la evidencia respecto de supervivencia global a 6 meses es entre $86,0 \%$ y $100 \%$; y de $59,0 \%$ a 24 meses (Schnitzbauer et al.; Alvarez et al, 2013; Schadde et al., 2014b; Hernandez-Alejandro et al.).

\section{DISCUSIÓN}

Los resultados reportados con ALPPS parecen ser superiores para casos de metástasis hepáticas de origen colorrectal (Schadde et al., 2014b; Hernandez-Alejandro et al.). Es por ello que existe además, evidencia del uso de neoadyuvancia con quimioterapia (previa al ALPPS), lo que ha permitido evaluar además evaluar la biología tumoral.

Dada la evidencia existente respecto de morbilidad y mortalidad asociada (incluida aquella relacionada con la necesidad de realizar reconstrucciones biliares (Schnitzbauer et al.; Li et al ., 2013; Nadalin et al.), el ALPPS debiera reservarse solamente para pacientes que requieren una hepatectomía en dos etapas, pero cuyo remanente hepático sea inferior al $25 \%$ a $30 \%$; y considerar como contraindicaciones relativas un bajo performance status y sujetos mayores de 70 años de edad (Bertens et al.). 
Por otra parte, se han reportado variaciones a la técnica original. Existe evidencia que un porcentaje relevante de las complicaciones se atribuyen a fístulas biliares, razón por la que la ligadura del conducto hepático derecho durante la primera etapa del procedimiento no debiera utilizarse. Finalmente, se ha verificado que la esqueletización del pedículo hepático de tejido linfonodal puede facilitar la devascularización del conducto biliar, por lo que debe ser observado con cautela en casos en que no sea relevante su utilización.

En resumen, ALPPS es una técnica novedosa para la resección de tumores malignos primarios y metastásicos del hígado; que ofrece a pacientes considerados no resecables, una oportunidad de tratamiento. Sin embargo, la elevada morbimortalidad, asociada no sólo a la gravedad y gran variabilidad de los pacientes, sino que también a algunas variantes técnicas; sugiere la necesidad de una selección rigurosa de los casos, puesto que la reproducción de resultados es difícil. Por esta razón, se hace necesario generar un protocolo estandarizado, que permita entre otras cosas, poder comparar realmente los resultados.

MANTEROLA, C. \& OTZEN, T. Associating liver partition and portal vein ligation for staged hepatectomy (ALPPS). Int. J. Morphol., 35(3):1083-1090, 2017.

SUMMARY: Postoperative hepatic malfunction subsequent to insufficiency of hepatic remnant is a complex and dire problem in patients subjected to large hepatic resections. The associating liver partition and portal vein ligation for staged hepatectomy (ALPPS), is a novel approach for oncology patients whose hepatic tumors were previously considered non-resectable. The technique is performed in two phases. The first one comprises the ligation of the right portal vein branch. Subsequently, a parenchymal transection is performed, including or not, the middle hepatic vein. A plastic bag is employed to cover the tumoral liver, and the abdomen is closed. The second one is performed at 7 to 15 days interval. After laparotomy, the plastic bag is removed. The right artery, bile duct and hepatic vein are sectioned and the tumoral liver is removed. Drain was placed at the resection surface, and the abdomen is closed. ALPPS can enable curative resection of hepatic metastasis in patients with tumors previously considered nonresectable. The aim of this manuscript was to describe the indications and technical aspects of ALPPS in relation to the first case carried out in our city, in a 47-year-old woman with advanced gallbladder cancer with bilobar metastases.

KEY WORDS: "Liver Neoplasms"[Mesh]; "Liver Neoplasms/surgery"[Mesh]; "Neoplasm Metastasis"'[Mesh]; "Neoplasm Metastasis/surgery"[Mesh]; "Hepatectomy"[Mesh]; "Gallbladder Neoplasms"[Mesh]) AND "Gallbladder Neoplasms/surgery"[Mesh]; "Liver Failure, Acute"[Mesh]; "Liver Regeneration"[Mesh]; ALPPS; non-colorectal liver metastases;.

\section{REFERENCIAS BIBLIOGRÁFICAS}

Abulkhir, A.; Limongelli, P.; Healey, A. J.; Damrah, O.; Tait, P.; Jackson, J.; Habib, N. \& Jiao, L. R. Preoperative portal vein embolization for major liver resection: a meta-analysis. Ann. Surg., 247(1):49-57, 2008.

Adam, R.; Imai, K.; Castro Benitez, C.; Allard, M. A.; Vibert, E.; Sa Cunha, A.; Cherqui, D.; Baba, H. \& Castaing, D. Outcome after associating liver partition and portal vein ligation for staged hepatectomy and conventional two-stage hepatectomy for colorectal liver metastases. $\mathrm{Br}$. J. Surg., 103(11):1521-9, 2016.

Alvarez, F. A.; Ardiles, V.; de Santibañez, M.; Pekolj, J. \& de Santibañes, E. Associating liver partition and portal vein ligation for staged hepatectomy offers high oncological feasibility with adequate patient safety: a prospective study at a single center. Ann. Surg., 261(4):72332, 2015.

Alvarez, F. A.; Ardiles, V.; Sánchez Claria, R.; Pekolj, J. \& de Santibañez, E. Associating liver partition and portal vein ligation for staged hepatectomy (ALPPS): tips and tricks. J. Gastrointest. Surg., 17(4):81421, 2013.

Baumgart, J.; Lang, S. \& Lang, H. A new method for induction of liver hypertrophy prior to right trisectionectomy: A report of three cases. HPB (Oxford), 13(Suppl. 2):71-2, 2011.

Bertens, K. A.; Hawel, J.; Lung, K.; Buac, S.; Pineda-Solis, K. \& HernandezAlejandro, R. ALPPS: challenging the concept of unresectability--a systematic review. Int. J. Surg., 13:280-7, 2015.

Björnsson, B.; Sparrelid, E.; Hasselgren, K.; Gasslander, T.; Isaksson, B. \& Sandström, P. Associating liver partition and portal vein ligation for primary hepatobiliary malignancies and non-colorectal liver metastases. Scand. J. Surg., 105(3):158-62, 2016a.

Björnsson, B.; Sparrelid, E.; Røsok, B.; Pomianowska, E.; Hasselgren, K.; Gasslander, T.; Bjørnbeth, B. A.; Isaksson, B. \& Sandström, P. Associating liver partition and portal vein ligation for staged hepatectomy in patients with colorectal liver metastases--Intermediate oncological results. Eur. J. Surg. Oncol., 42(4):531-7, 2016 b.

Buac, S.; Schadde, E.; Schnitzbauer, A. A.; Vogt, K. \& Hernandez-Alejandro, R. The many faces of ALPPS: surgical indications and techniques among surgeons collaborating in the international registry. $H P B$ (Oxford), 18(5):442-8, 2016.

Cai, Y. L.; Song, P. P.; Tang, W. \& Cheng, N. S. An updated systematic review of the evolution of ALPPS and evaluation of its advantages and disadvantages in accordance with current evidence. Medicine (Baltimore), 95(24):e3941, 2016.

Capussotti, L.; Muratore, A.; Baracchi, F.; Lelong, B; Ferrero, A.; Regge, D. \& Delpero, J. R. Portal vein ligation as an efficient method of increasing the future liver remnant volume in the surgical treatment of colorectal metastases. Arch. Surg., 143(10):978-82, 2008.

Chan, A. C.; Poon, R. T. \& Lo, C. M. Modified Anterior Approach for the ALPPS Procedure: How We Do It. World J. Surg., 39(11):2831-5, 2015.

Clavien, P. A.; Barkun, J.; de Oliveira, M. L.; Vauthey, J. N.; Dindo, D.; Schulick, R. D.; de Santibañes, E.; Pekolj, J.; Slankamenac, K.; Bassi, C.; Graf, R.; Vonlanthen, R.; Padbury, R.; Cameron, J. L. \& Makuuchi, M. The Clavien-Dindo classification of surgical complications: fiveyear experience. Ann. Surg., 250(2):187-96, 2009.

Clavien, P. A.; Yadav, S.; Sindram, D. \& Bentley, R. C. Protective effects of ischemic preconditioning for liver resection performed under inflow occlusion in humans. Ann. Surg., 232(2):155-62, 2000.

de Graaf, W.; van den Esschert, J. W.; van Lienden, K. P. \& van Gulik, T. M. Induction of tumor growth after preoperative portal vein embolization: is it a real problem? Ann. Surg. Oncol., 16(2):423-30, 2009.

de Santibañes, E.; \& Clavien, P. R. Playing Play-Doh to prevent postoperative liver failure: the "ALPPS" approach. Ann. Surg., 255(3):415-7, 2012.

Donati, M.; Stavrou, G. A. \& Oldhafer, K. J. Current position of ALPPS in 
the surgical landscape of CRLM treatment proposals. World $J$. Gastroenterol., 19(39):6548-54, 2013.

Elias, D.; De Baere, T.; Roche, A; Mducreux; Leclere, J. \& Lasser, P. During liver regeneration following right portal embolization the growth rate of liver metastases is more rapid than that of the liver parenchyma. $B r$. J. Surg., 86(6):784-8, 1999.

Ettorre, G. M.; Guglielmo, N.; Felli, E.; Meniconi, R. L.; Colasanti, M.; Lepiane, P.; Santoro, R. \& Vennarecci, G. Is there still a room to improve the safety of ALPPS procedure? A new technical note. Eur. J. Sur g. Oncol., 41(11):1556-7, 2015.

Fukami, Y.; Kurumiya, Y. \& Kobayashi, S. Associating liver partition and portal vein ligation for staged hepatectomy (ALPPS): an analysis of tumor activity. Updates Surg., 66(3):223-5, 2014.

Gerard, A.; Buyse, M.; Pector, J. C.; Bleiberg, H.; Arnaud, J. P.; Willems, G.; Delvaux, G.; Lise, M.; Nitti, D. \& Depadt, G. Hepatic artery ligation with and without portal infusion of 5-FU. A randomized study in patients with unresectable liver metastases from colorectal carcinoma. The E.O.R.T.C. Gastrointestinal Cancer Cooperative Group (G.I. Group). Eur. J. Surg. Oncol., 17(3):289-94, 1991.

Hasselgren, K.; Sandström, P. \& Björnsson, B. Role of associating liver partition and portal vein ligation for staged hepatectomy in colorectal liver metastases: a review. World J. Gastroenterol., 21(15):4491-8, 2015.

Hernandez-Alejandro, R.; Bertens, K. A.; Pineda-Solis, K. \& Croome, K. P. Can we improve the morbidity and mortality associated with the associating liver partition with portal vein ligation for staged hepatectomy (ALPPS) procedure in the management of colorectal liver metastases? Surgery, 157(2):194-201, 2015.

Kawaguchi, D.; Hiroshima, Y.; Matsuo, K.; Endo, I.; Koda, K. \& Tanaka, K. Parenchymal congestion is important for rapid regeneration of the future liver remnant following the ALPPS procedure. Anticancer Res. 36(9):4731-8, 2016

Knoefel, W. T.; Gabor, I.; Rehders, A.; Alexander, A.; Krausch, M.; Schulte am Esch, J.; Fürst, G. \& Topp, S. A. In situ liver transection with portal vein ligationfor rapid growth of the future liver remnant in two-stage liver resection. Br. J. Surg., 100(3):388-94, 2013.

Kremer, M.; Manzini, G.; Hristov, B.; Polychronidis, G.; Mokry, T.; Sommer, C. M.; Mehrabi, A.; Weitz, J.; Büchler, M. W. \& Schemmer, P. Impact of neoadjuvant chemotherapy on hypertrophy of the future liver remnant after associating liver partition and portal vein ligation for staged hepatectomy. J. Am. Coll. Surg., 221(3):717-728.e1, 2015.

Lang, S. A.; Loss, M.; Benseler, V.; Glockzin, G. \& Schlitt, H. J. Longterm results after in-situ split (ISS) liver resection. Langenbecks Arch. Surg., 400(3):361-9, 2015.

Li, J.; Girotti, P.; Königsrainer, I.; Ladurner, R.; Königsrainer, A. \& Nadalin, S. ALPPS in right trisectionectomy: a safe procedure to avoid postoperative liver failure? J. Gastrointest. Surg., 17(5):956-61, 2013.

Linecker, M.; Stavrou, G. A.; Oldhafer, K. J.; Jenner, R. M.; Seifert, B.; Lurje, G.; Bednarsch, J.; Neumann, U.; Capobianco, I.; Nadalin, S.; Robles-Campos, R.; de Santibañes, E.; Malagó, M.; Lesurtel, M.; Clavien, P. A. \& Petrowsky, H. The ALPPS Risk Score: Avoiding futile use of ALPPS. Ann. Surg., 264(5):763-71, 2016.

Makuuchi M.; Thai, B. L.; Takayasu, K.; Takayama, T.; Kosuge, T.; Gunvén, P.; Yamazaki, S.; Hasegawa, H. \& Ozaki, H. Preoperative portal embolization to increase safety of major hepatectomy for hilar bile duct carcinoma: a preliminary report. Surgery, 107(5):521-7, 1990.

Mann, D. V.; Lam, W. W.; Hjelm, N. M.; So, N. M.; Yeung, D. K.; Metreweli, C. \& Lau, W. Y. Human liver regeneration: hepatic energy economy is less efficient when the organ is diseased. Hepatology, 34(3):557-65, 2001.

Margarit, C. Prevención de la insuficiencia hepática aguda tras la resección hepática. GH Continuada, 4(1):37-48, 2005.

Nadalin, S.; Capobianco, I.; Li, J.; Girotti, P.; Königsrainer, I. \& Königsrainer, A. Indications and limits for associating liver partition and portal vein ligation for staged hepatectomy (ALPPS). Lessons Learned from 15 cases at a single centre. Z. Gastroenterol., 52(1):3542, 2014.
Oldhafer, K. J.; Donati, M.; Jenner, R. M.; Stang, A. \& Stavrou, G. A. ALPPS for patients with colorectal liver metastases: effective liver hypertrophy, but early tumor recurrence. World J. Surg., 38(6):1504-9, 2014

Rahbari, N. N.; Garden, O. J.; Padbury, R.; Brooke-Smith, M.; Crawford, M.; Adam, R.; Koch, M.; Makuuchi, M.; Dematteo, R. P.; Christophi, C.; Banting, S.; Usatoff, V.; Nagino, M.; Maddern, G.; Hugh, T. J.; Vauthey, J. N.; Greig, P.; Rees, M.; Yokoyama, Y.; Fan, S. T.; Nimura, Y.; Figueras, J.; Capussotti, L.; Büchler, M. W. \& Weitz, J. Posthepatectomy liver failure: a definition and grading by the International Study Group of Liver Surgery (ISGLS). Surgery, 149(5):713-24, 2011.

Ratti, F.; Cipriani, F.; Gagliano, A.; Catena, M.; Paganelli, M. \& Aldrighetti, L. Defining indications to ALPPS procedure: technical aspects and open issues. Updates Surg., 66(1):41-9, 2014.

Ribero, D.; Abdalla, E. K.; Madoff, D. C.; Donadon, M.; Loyer, E. M. \& Vauthey, J. N. Portal vein embolization before major hepatectomy and its effects on regeneration, resectability and outcome. Br. J. Sur g., 94(11):1386-94, 2007.

Robles Campos, R.; Brusadin, R.; López Conesa, A. \& Parrilla Paricio, P. Resección en 2 tiempos de tumores hepáticos perihiliares con torniquete en la cisura umbilical y embolización portal secuencial al cuarto día postoperatorio (ALTPS modificado). Cir. Esp., 92(10):682-6, 2014.

Robles, R.; Marín, C.; López-Conesa, A.; Capel, A.; Pérez-Flores, D. \& Parrilla, P. Comparative study of right portal vein ligation versus embolisation for induction of hypertrophy in two-stage hepatectomy for multiple bilateral colorectal liver metastases. Eur. J. Surg. Oncol., 38(7):586-93, 2012.

Rochet, S.; Leiva Espinoza, J.; Álvarez, F.; Goransky, J.; de Santibañes, M.; Ardiles, V. \& de Santibañes, E. Asociación de partición hepática y ligadura portal para hepatectomía diferida (ALPPS) en pacientes con enfermedad hepática avanzada. Rev. Argent. Cirug., 106(1):19-30, 2014.

Røsok, B. I.; Björnsson, B.; Sparrelid, E.; Hasselgren, K.; Pomianowska, E.; Gasslander, T.; Bjørnbeth, B. A.; Isaksson, B. \& Sandström, P. Scandinavian multicenter study on the safety and feasibility of the associating liver partition and portal vein ligation for staged hepatectomy procedure. Surgery, 159(5):1279-86, 2016.

Sala, S.; Ardiles, V.; Ulla, M.; Alvarez, F.; Pekolj, J. \& de Santibañes, E. Our initial experience with ALPPS technique: encouraging results. Updates Surg., 64(3):167-72, 2012.

Schadde, E.; Ardiles, V.; Robles-Campos, R.; Malago, M.; Machado, M.; Hernandez-Alejandro, R.; Soubrane, O.; Schnitzbauer, A. A.; Raptis, D.; Tschuor, C.; Petrowsky, H.; De Santibanes, E.; Clavien, P. A. \& ALPPS Registry. Early survival and safety of ALPPS: first report of the International ALPPS Registry. Ann. Surg., 260(5):829-36, 2014b.

Schadde, E.; Ardiles, V.; Slankamenac, K.; Tschuor, C.; Sergeant, G.; Amacker, N.; Baumgart, J.; Croome, K.; Hernandez-Alejandro, R.; Lang, H.; de Santibañes, E. \& Clavien, P. A. ALPPS offers a better chance of complete resection in patients with primarily unresectable liver tumors compared with conventional-staged hepatectomies: results of a multicenter analysis. World J. Surg., 38(6):1510-9, 2014a.

Schnitzbauer, A. A.; Lang, S. A.; Goessmann, H.; Nadalin, S.; Baumgart, J.; Farkas, S. A.; Fichtner-Feigl, S.; Lorf, T.; Goralcyk, A.; Hörbelt, R.; Kroemer, A.; Loss, M.; Rümmele, P.; Scherer, M. N.; Padberg, W.; Königsrainer, A.; Lang, H.; Obed, A. \& Schlitt, H. J. Right portal vein ligation combined with in situ splitting induces rapid left lateral liver lobe hypertrophy enabling 2-staged extended right hepatic resection in small-for-size settings. Ann. Surg., 255(3):405-14, 2012.

Shindoh, J.; Vauthey, J. N.; Zimmitti, G.; Curley, S. A.; Huang, S. Y.; Mahvash, A.; Gupta, S.; Wallace, M. J. \& Aloia, T. A. Analysis of the efficacy of portal vein embolization for patients with extensive liver malignancy and very low future liver remnant volume, including a comparison with the associating liver partition with portal vein ligation for staged hepatectomy approach. J. Am. Coll. Surg., 217(1):126-33, 2013.

Tanaka, K.; Kikuchi, Y.; Kawaguchi, D.; Murakami, T.; Hiroshima, Y. \& 
Matsuo, K. Modified ALPPS procedures avoiding division of portal pedicles. Ann. Surg., 265(2):e14-e20, 2017.

Tanaka, K.; Matsuo, K.; Murakami, T.; Kawaguchi, D.; Hiroshima, Y.; Koda, K.; Endo, I.; Ichikawa, Y.; Taguri, M. \& Tanabe, M. Associating liver partition and portal vein ligation for staged hepatectomy (ALPPS): shortterm outcome, functional changes in the future liver remnant, and tumor growth activity. Eur. J. Surg. Oncol., 41(4):506-12, 2015.

Torres, O. J.; Moraes-Junior, J. M.; Lima e Lima, N. C. \& Moraes, A. M. Associating liver partition and portal vein ligation for staged hepatectomy (ALPPS): a new approach in liver resections. Arq. Bras. Cir. Dig., 25(4):290-2, 2013.

Truant, S.; Scatton, O.; Dokmak, S.; Regimbeau, J.M.; Lucidi, V.; Laurent, A.; Gauzolino, R.; Castro Benitez, C.; Pequignot, A.; Donckier, V.; Lim, C.; Blanleuil, M. L.; Brustia, R.; Le Treut, Y. P.; Soubrane, O.; Azoulay, D.; Farges, O.; Adam, R.; Pruvot, F. R. \& e-HPBchir Study Group from the Association de Chirurgie Hépato-Biliaire et de Transplantation (ACHBT). Associating liver partition and portal vein ligation for staged hepatectomy (ALPPS): impact of the inter-stages course on morbimortality and implications for management. Eur. J. Sur g. Oncol., 41(5):674-82, 2015

Uribe, M. M.; Uribe-Echevarría, A. S.; Núñez, V. J.; Martínez, M. W.; Paqui, M. L.; Camacho, M. J. P. \& Romanque, U. P. Associating liver partition and portal vein ligation for staged hepatectomy, a new hepatic procedure. Rev. Chil. Cir., 67:185-90, 2015.

Vivarelli, M.; Vincenzi, P.; Montalti, R.; Fava, G.; Tavio, M.; Coletta, M.; Vechhi, A.; Nicolini, D.; Agostini, A.; Ali Ahmed, E.; Giovagnoni, A. $\&$ Mocchegiani, F. ALPPS Procedure for extended liver resections: A single centre experience and a systematic review. PLoS One, 10(12):e0144019, 2015.

Yigitler, C.; Farges, O.; Kianmanesh, R.; Regimbeau, J. M.; Abdalla, E. K. $\&$ Belghiti, J. The small remnant liver after major liver resection: how common and how revelant? Liver Transpl., 9(9):S18-25, 2003.

Zhang, G. Q.; Zhang, Z. W.; Lau, W. Y. \& Chen, X. P. Associating liver partition and portal vein ligation for staged hepatectomy (ALPPS): a new strategy to increase resectability in liver surgery. Int. J. Sur g., 12(5):437-41, 2014

\author{
Dirección para correspondencia: \\ Dr. Carlos Manterola \\ Departamento de Cirugía y CEMyQ \\ Universidad de La Frontera \\ Temuco \\ CHILE
}

E-mail: carlos.manterola@ufrontera.cl

Recibido : 11-03-2017

Aceptado: 25-05-2017 